\title{
Paraganglioma de corpo aórtico em cão
}

\author{
Aortic Body Paraganglioma in Dog
}

\begin{abstract}
Paulo Henrique Leal Bertolo', Lucien Roberta Valente Miranda de Aguirra², Suellen da Gama Barbosa Monger², Adriana Maciel de Castro Cardoso ${ }^{2}$, Rosemeri de Oliveira Vasconcelos' \& Washington Luiz Assunção Pereira ${ }^{2}$
\end{abstract}

\begin{abstract}
Background: Aortic body paragangliomas are uncommon neoplasms that develop mainly in aortic and carotid bodies. It has been supposed that genetic factors and chronic hypoxia may stimulate tumor development. The brachycephalic dog breeds, as Boxer, are most predisposed to present this neoplasm. The clinical symptomatology is related to tumor size and localization. Usually aortic body paraganglioma has benign biological behavior, when it is malignant, rarely promotes metastases. The aim of this study was to report a case of the aortic body paraganglioma as death cause in a dog.

Case: A canine, 10-year-old, male, cross breed, presented clinical signs as anorexia, emesis, cough, dyspnea and exercise intolerance. After death the animal was examined at the Department of Veterinary Pathology at the Federal Rural University of Amazonia. On necropsy, no pericardial effusion was identified, however pleural and abdominal effusion was observed, volume like 1000 and $700 \mathrm{~mL}$, respectively. The heart had a neoplasm near the left atrium, it measured $6.5 \times 8.2 \mathrm{~cm}$, had irregular surface, firm consistency, grayish color, and at the cut showed infiltration in the myocardium, as well as obstruction of the left atrial lumen and left ventricle concentric hypertrophy. No distant metastases were found. Microscopically, the tumor consisted of polyhedral morphology cells, eosinophilic cytoplasm, spherical and hyperchromatic nucleus. Cells were grouped into lobes separated by fibrovascular stroma, large cells (less uniform cells), low mitotic rate and myocardial infiltration. On immunohistochemical analysis anti-cytokeratin, anti-vimentin and anti-S-100 antibodies were used. Tumour cells stained was absent for anti-cytokeratin and anti-vimentin, but was anti-S-100 positive. A case of malignant aortic body paraganglioma grade II was diagnosed.

Discussion: The reported case was diagnosed in cross breed dog. However, brachycephalic dog breeds, as Boxer, are more predisposed to develop this kind of tumor. The development of these tumors is related to the genetic factors and chronic hypoxia. It was suggested that the tumor origin on this case is related to genetic factors, because the animal had no respiratory diseases, he was not brachycephalic and lived in a low altitude city, what exclude the possibility to have been induced by chronic hypoxia imposed by low oxygen. The clinical symptomatology presented by the animal days before death was related to the localization and size of the tumor. The macroscopic findings were similar to those of other studies. And the histopathological findings of the report were indicative for the histological classification of malignant aortic body paraganglioma. On immunohistochemical analysis it was negative for anti-cytokeratin and anti-vimentin antibodies, however was positive for anti-S-100 antibody, thus differentiated from other tumors that may occur in this local and confirmed the diagnosis of malignant aortic body paraganglioma grade II. The dog died due to tumor-related causes, however some authors indicate de tumor as a necropsy finding. The findings made it possible to conclude that tumor was aortic body paraganglioma and that it promoted cardiorespiratory complications related to localization and infiltration, what was worsened by pleural effusion leading the animal to the cardiogenic shock that culminated with death. It shows the importance of including this tumor in the list of differential diagnoses of heart diseases in dogs.
\end{abstract}

Keywords: canine, veterinary oncology, veterinary cardiology, cardiac neoplasm, chemodectoma.

Descritores: canino, oncologia veterinária, cardiologia veterinária, neoplasia cardíaca, quimiodectoma.

${ }^{1}$ Departamento de Patologia Veterinária, Faculdade de Ciências Agrárias e Veterinárias, Universidade Estadual Paulista (FCAV/UNESP), Jaboticabal, SP, Brazil. ${ }^{2}$ Setor de Patologia Veterinária, Instituto da Saúde e Produção Animal, Universidade Federal Rural da Amazônia (ISPA/UFRA), Belém, PA, Brazil. CORRESPONDENCE: P.H.L. Bertolo [henrique_medvet@ yahoo.com.br - Tel.: +55 (16) 98103-8678]. Departamento de Patologia Veterinária - FCAV/UNESP. Via de Acesso Prof. Paulo Donato Castellane s/n. CEP 14884-900 Jaboticabal, SP, Brazil. 


\section{INTRODUÇÃO}

Os paragangliomas de corpo aórtico são neoplasias raras que se desenvolvem principalmente nos corpos aórticos e carotídeos [9]. Tem sido sugerido que a predisposição genética agravada por hipóxia crônica pode incitar o desenvolvimento do tumor [6,7], sendo os cães braquicefálicos, como o boxer, os mais predispostos a essa neoplasia $[8,11]$.

A sintomatologia clínica está relacionada com a localização e tamanho do tumor [10]. Como se localizam na base do coração, pode comprometer a função cardíaca por comprimir os vasos da base do coração e por infiltrar no miocárdio [3,10,14].

Comumente o comportamento biológico do paraganglioma de corpo aórtico é benigno, e quando malignos raramente promovem metástases [9], sendo classificados em três graus de acordo com as características como indice mitótico, o padrão de crescimento invasivo, pleomorfismo celular e metástase a distância [2]. Diante do exposto, objetivou descrever um caso de paraganglioma de corpo aórtico como causador do óbito em cão.

\section{CASO}

Um canino, sem raça definida, macho, com 10 anos de idade, com histórico clínico de anorexia, êmese, tosse, dispneia e intolerância a exercícios veio a óbito e foi encaminhado ao Setor de Patologia Veterinária da Universidade Federal Rural da Amazônia, Belém-PA, para análise necroscópica.

Na necropsia, não foi identificada efusão pericárdica, porém observou-se efusão pleural e abdominal num volume aproximado de 1000 e 700 mililitros, respectivamente. $O$ coração exibiu massa junto ao átrio esquerdo, medindo $6,5 \times 8,2 \mathrm{~cm}$, de superfície irregular, consistência firme, coloração acinzentada (Figura 1A), e ao corte apresentou infiltração no miocárdio, bem como obstrução do lúmen atrial esquerdo e hipertrofia concêntrica do ventrículo esquerdo (Figura 1B). Não foram encontradas metástases à distância.

Microscopicamente, o tumor era constituído por células de morfologia poliédrica, com citoplasma eosinofílico, núcleos esféricos e hipercromáticos. As células estavam agrupadas em lóbulos separados por estroma fibrovascular bastante desenvolvido, foram também visualizadas células destacadamente grandes (células pouco uniformes), baixo índice mitótico e infiltração no miocárdio (Figura 2A).
Na análise imuno-histoquímica foram utilizados anticorpos anti-citoqueratina ${ }^{1}$ 1:200 (clone AE1/AE3, Dako M3515), anti-vimentina ${ }^{2}$ 1:150 (clone V9, Dako M0725) e anti- S-100 1:400 (Poyclonal Rabbit, Dako Z0628) por meio do complexo estreptavidina-biotina- peroxidase $^{1}$ (kit LSAB+System HRP, Dako, Cód. K0690-1). A recuperação antigênica foi feita pelo calor em câmara de pressão Pascal ${ }^{1}$ (Dako, modelo S2800) com solução tampão de citrato de sódio $\mathrm{pH}=6$. A inibição das reações inespecíficas foi realizada por meio de bloqueio duplo, com solução de metanol + peróxido de hidrogênio a $10 \%$ e com produto comercial ${ }^{1}$ (Protein Block, Dako X0909). Como cromógeno utilizou-se DAB $^{1}$ (3-3'diaminobenzidina, Dako K3468) e os cortes do tumor foram contra corados com hematoxilina de Harris. A imunomarcação foi ausente, nas células neoplásicas, para os anticorpos anti-citoqueratina e anti-vimentina, porém foi positiva moderada e difusa no citoplasma das células neoplásicas para o anticorpo anti-S-100 (Figura 2B). Segundo os achados anatomopatológico, histopatológico e a associação do histórico clínico, adicionalmente, a imunomarcação positiva para proteína S-100, foi configurado o diagnóstico de paraganglioma de corpo aórtico maligno grau II.

\section{DISCUSSÃO}

O caso ocorreu em canino, sem raça definida, de dez anos de idade. No entanto, os cães braquicefálicos como o boxer são mais propensos ao desenvolvimento desse tumor. Enquanto a idade correspondeu à faixa etária média das ocorrências que é entre oito e treze anos $[8,11]$.

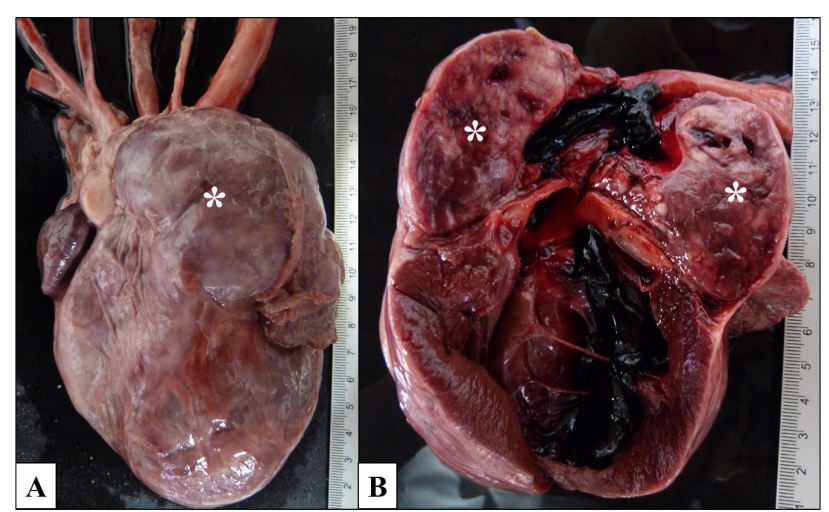

Figura 1. Avaliação macroscópica do coração do cão. A- Coração exibiu massa junto ao átrio esquerdo, medindo $6,5 \times 8,2 \mathrm{~cm}$, de superfície irregular, consistência firme, coloração acinzentada (Asterisco). B- Ao corte apresentou infiltração no miocárdio, além de obstrução do lúmen atrial esquerdo (Asteriscos) e hipertrofia concêntrica do ventrículo esquerdo. 


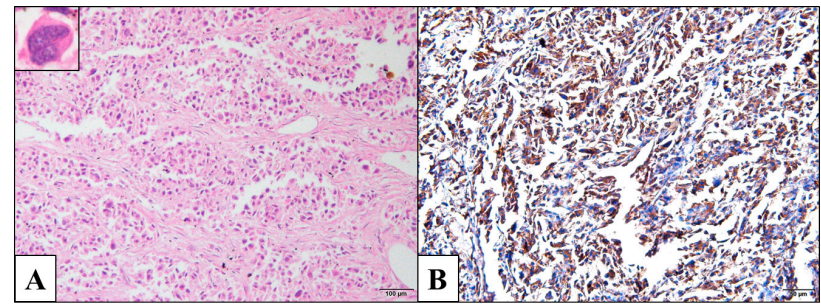

Figura 2. Avaliação histopatológica e imuno-histoquímica do tumor. A- Microscopicamente o tumor apresentou células de morfologia poliédrica, com citoplasma eosinofílico, núcleos esféricos e hipercromáticos. As células estavam agrupadas em lóbulos separados por estroma fibrovascular, foram também visualizadas células destacadamente grandes (células pouco uniformes), baixo índice mitótico e infiltração no miocárdio [HE, 40x]. B- Imunomarcação positiva moderada e difusa no citoplasma das células neoplásicas para o anticorpo anti-S-100 [40x].

O desenvolvimento desses tumores está relacionado à predisposição genética agravada por hipóxia crônica, e este esforço respiratório pode ser causado por enfermidades, conformação do trato respiratório, como a dos cães braquicefálicos, e altitudes elevadas [6,7]. No entanto, sugere-se que a origem do paraganglioma de corpo aórtico do caso relatado tenha relação com fatores genéticos, visto que o animal não apresentava enfermidades respiratórias, não era braquicefálico e residia numa cidade com altitude de apenas dez metros, excluindo a possibilidade de ter sido induzido por hipóxia crônica imposta por baixa tensão de oxigênio.

A sintomatologia clínica apresentada pelo animal dias antes do óbito, que está relacionada à localização e tamanho do tumor, foi similar aos sinais clínicos de estudos anteriores a este $[5,8,12,13]$. Os achados macroscópicos relacionados às características do tumor como consistência, coloração, infiltração local e obstrução do átrio esquerdo já tinham sido descritos anteriormente [10].

Os achados histopatológicos do relato são indicativos para a classificação histológica do paraganglioma de corpo aórtico maligno [2,9]. A reação imuno-histoquímica foi negativa para os anticorpos anti-citoqueratina e anti-vimentina, resultados importantes para o diagnóstico diferencial de tumores que podem ocorrer nessa região como o carcinoma ectópico de tireóide, linfoma e hemangiossarcoma [8]. A imunomarcação citoplasmática para o anticorpo S-100 confirma o diagnóstico de paraganglioma de corpo aórtico. A marcação para S-100 pode indicar um grau de malignidade menor [1]. Apesar da maioria desses tumores serem benignos, o tumor relatado foi classificado como maligno de grau II segundo a classificação proposta por Brown et al. [2].

No caso relatado não foram encontradas metástases à distância, corroborando com a literatura veterinária que enfatiza potencial metastático raro desse tumor [9]. No entanto, já foram relatados casos de paraganglioma de corpo aórtico metastáticos em pulmão e baço [4], pulmão e rins [1] e somente em pulmão $[13,15]$. O animal veio a óbito devido causas relacionadas ao tumor, assim como foi observado anteriormente [10,15]. Entretanto, Garrido et al. [5] não fizeram essa correlação considerando o tumor um achado incidental de necropsia.

Os achados anatomopatológicos permitiram concluir que o tumor tratava-se de um paraganglioma de corpo aórtico, e que este tumor promoveu complicações cardiorespiratórias devido à localização e infiltração, sendo agravada pela efusão pleural levando o animal ao quadro de choque cardiogênico que culminou com o óbito do mesmo, demonstrando a importância de incluir este tumor na lista de diagnósticos diferenciais de cardiopatias em cães.

\section{MANUFACTURERS}

${ }^{1}$ Dako North America Inc. Carpinteria, CA, USA.

${ }^{2}$ Dako Denmark S/A. Glostrup, Denmark.

Declaration of interest. The authors report no conflicts of interest. The authors alone are responsible for the content and writing of the paper.

\section{REFERENCES}

1 Aresu L., Tursi M., Iussich S., Guarda F. \& Valenza F. 2006. Use of S-100 and Chromogranin A Antibodies as Immunohistochemical Markers on Detection of Malignancy in Aortic Body Tumors in Dog. Journal of Veterinary Medical Science. 68(11): 1229-1233.

2 Brown P.J., Rema A. \& Gartner F. 2003. Immunohistochemical characteristics of canine aortic and carotid body tumours. Journal Veterinary Medicine. 50(3): 140-144.

3 Cavalcanti G.A.O., Muzzi R.A.L., Bezerra Júnior P.S., Nogueira R.B. \& Varaschin M.S. 2006. Fibrilação atrial em cão associada ao quimiodectoma infiltrativo atrial: relato de caso. Arquivo Brasileiro de Medicina Veterinária e Zootecnia. 58(6): 1043-1047. 
4 Chiquito M., Werner P.R. \& Pachaly J.R. 1998. Ocorrência de quimiodectomas em animais no primeiro planalto do Paraná, Brasil. Arquivo de Ciências Veterinárias e Zoologia da UNIPAR. 1(1): 67-70.

5 Garrido E., Jacintho A.P.P., Magalhães G.M., Cândido E.M. \& Vasconcelos R.O. 2008. Quimiodectoma em cão: relato de caso. Veterinária e Zootecnia. 15(Supl 2): 49-50.

6 Hadlow W.J. 1986. Carotid body tumor: an incidental finding in older ranch mink. Veterinary Pathology. 23(2): 162-169.

7 Hayes H.M. 1975. An hypothesis for the aetiology of canine chemoreceptor system neoplasms, based upon an epidemiological study of 73 cases among hospital patients. Journal of Small Animal Practice. 16(5): 337-343.

8 Mesquita L.P., Abreu C.C., Nogueira C.I., Wouters A.T.B., Wouters F., Bezerra Júnior P.S., Muzzi R.A.L. \& Varaschin M.S. 2012. Prevalência e aspectos anatomopatológicos das neoplasias primárias do coração, de tecidos da base do coração e metastáticas, em cães do sul de minas gerais (1994-2009). Pesquisa Veterinária Brasileira. 32(11): $1155-1163$.

9 Miller L.M., Van Vleet J.F. \& Gal A. 2013. Sistema cardiovascular e vasos linfáticos. In: Zachary J.F. \& Mcgavin M.D. (Eds). Bases da Patologia em Veterinária. 5.ed. Rio de Janeiro: Elsevier, pp.542-591.

10 Moura V.M.B.D., Goiozo P.F.I., Thomé H.E., Caldeira C.P. \& Bandarra E.P. 2006. Quimiodectoma como causa de morte súbita em cão - relato de caso. Veterinária Notícias. 12(1): 95-99.

11 Noszczyk-Nowak A., Nowak M., Paslawska U., Atamaniuk W. \& Nicpon J. 2010. Cases with manifestation of chemodectoma diagnosed in dogs in department of internal diseases with horses, dogs and cats clinic, veterinary medicine faculty, university of environmental and life sciences, wroclaw, poland. Acta Veterinaria Scandinavica. 52(32): 1-7.

12 Rajagopalan V., Jesty S.A., Craig L.E. \& Gompf R. 2013. Comparison of presumptive echocardiographic and definitive diagnoses of cardiac tumors in dogs. Journal of Veterinary Internal Medicine. 27(5): 1092-1096.

13 Salomão M.C., Mattos A.S., Lucena A.R., Leite J.S., Mello M.F.V. \& Ferreira A.M.R. 2012. Tumor de arco aórtico em cão (Canis familiaris) - relato de caso. Veterinária e Zootecnia. 19(1): 107-109.

14 Sampaio R.L., Coelho H.E., Lacerda M.S., Oliveira P.L., Paro P.Z., Rezende R.S. \& Bittar J.F.F. 2008. Efusão pleural secundária por tumor de corpo aórtico em cães - relato de caso. Veterinária Notícias. 14(2): 55-62.

15 Zanoni D.S., Ferioli R.B., Xavier J.G., Sequeira J.L. \& Amorim R.F. 2016. Paraganglioma em cão associado à falência cardíaca: relato de caso. Veterinária e Zootecnia. 23(1): 72-77. 